\title{
Raman Microscopy: Three Variations on a Pharmaceutical Theme
}

\author{
F. C. Thorley ${ }^{*}$, G. A. Thomson ${ }^{*}$, K J Baldwin, D. C. Lee ${ }^{* *}$ and D. N. Batchelder ${ }^{*}$ \\ * Department of Physics and Astronomy, University of Leeds, Leeds LS2 9JT, UK \\ ${ }^{* *}$ GlaxoSmithKline, Medicines Research Centre, Stevenage, SG1 2NY, UK
}

The development of novel pharmaceuticals is driven by the requirement for high efficacy while retaining reduced side effects. This means that new medicines are very often relatively low dose (below $10 \% \mathrm{w} / \mathrm{w}$ drug substance in a tablet) and therefore they pose significant analytical challenges. Typical requirements include chemical identification of the drug substance, its crystalline form, the presence of any degradants and the distribution of drug substance and excipients.

Raman spectroscopy is becoming one of the favoured techniques for looking at tablets. Raman is a non-invasive and non-destructive technique that is very important in the pharmaceutical industry. In addition, with a Raman microscope it is possible to obtain spectra from particles of micrometer dimensions. This means that limits of detection can be lower for inhomogeneous samples than those of non-microscopic Raman techniques.

The most common Raman experiment for analysing tablets is the point-by-point mapping approach. This can be very time consuming and therefore difficult for trouble shooting or on-line analysis. One way of increasing the speed of analysis would be by increasing the laser power used (and thereby decreasing the acquisition time). This approach, however, increases the probability of causing chemical degradation.

We present three modifications to a Renishaw system 2000 Raman microscope that improve the microanalysis of pharmaceutical tablets by Raman microscopy. In the first, a diffractive optic splits the incoming laser beam into several spots, thus permitting a higher laser power. In the present experiment, the laser beam was split into four focal spots on the sample that were $10 \mu \mathrm{m}$ apart. The diffractive optic has been placed into the path of the beam of the Raman microscope as shown in Figure 1. This sampling approach allows the recording of several spectra simultaneously on the addressable charge-coupled device (CCD) and hence decreases the amount of time required to sample the tablet. In addition, the full confocality and spatial resolution of the Raman microscope are maintained.

In Raman studies of many samples, it is desirable to know the intensities of orthogonal, linearly-polarised components of the Raman bands. In the second modification, we have inserted a Rochon prism in the beam of Raman scattered light in the Raman microscope as shown in Figure 2. The prism splits the orthogonally polarised components of the Raman spectra into two separate beams that are recorded simultaneously using an addressable CCD. In addition to saving time, the simultaneous recording means that the components are sampled under identical experimental conditions.

In the direct imaging mode of the Raman microscope, the laser beam is defocused to illuminate an area on the sample that can be directly imaged by the scattered light onto the CCD camera.

A tuneable filter in the beam path removes all light from the scattered beam except that in a selected Raman band. Thus a Raman image of the illuminated area is formed on the CCD. This type of imaging can produce detailed composition maps of a sample in a much shorter time than point-by-point mapping. We have modified the optical arrangement in the Raman microscope by placing the tuneable filter between positive and negative lenses as shown in Figure 3 . The principal rays from different points on the sample now pass through the filter at the same angle. This change significantly improved the uniformity of the intensity of the Raman image over the sample.

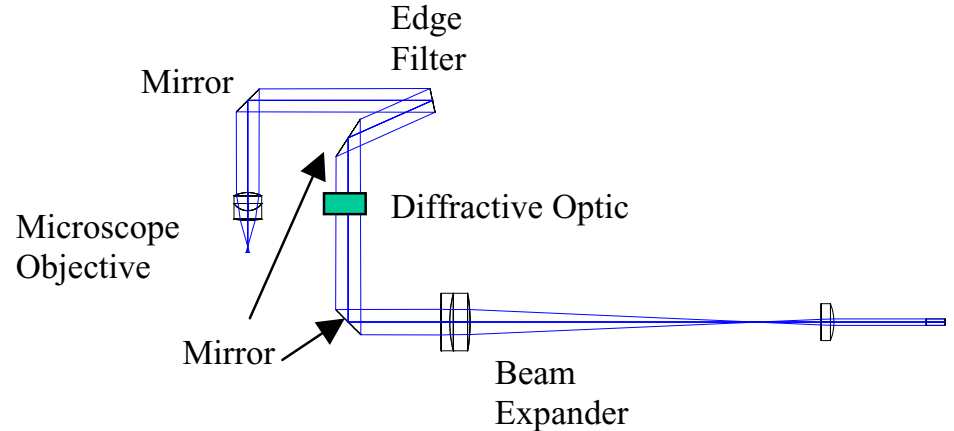


FIG. 1. Schematic diagram showing the location of the diffractive optic in the Raman

microscope

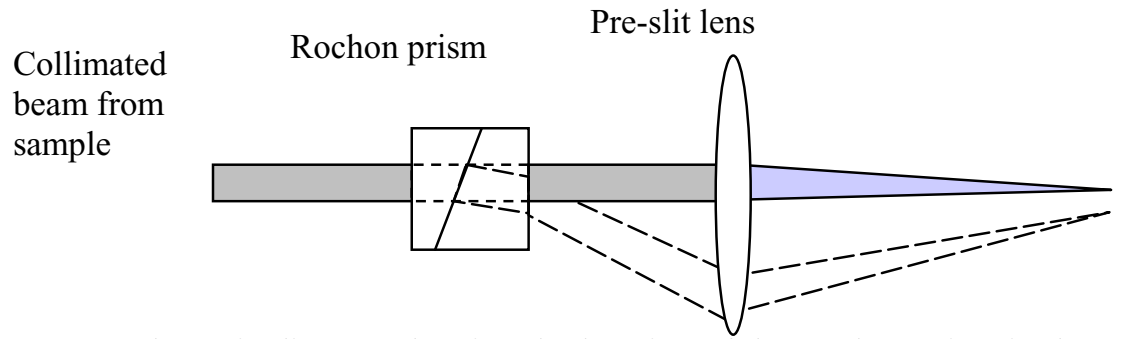

FIG. 2. Schematic diagram showing the location of the Rochon prism in the Raman separate foci of the orthogonally polarised beams at the entrance slit of the spectrometer

microscope and the two

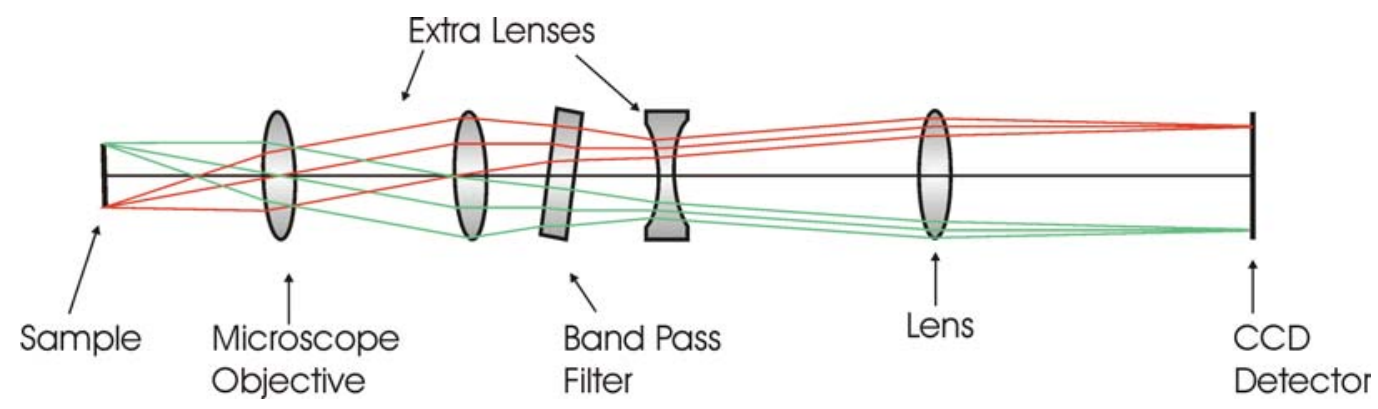

FIG. 3. Schematic diagram of the new optical arrangement for direct Raman imaging 